\title{
Research on WSN Double-Radius Localization Algorithm Based on Partition Judgment Mechanism
}

\author{
Jijun Zhao, Hua Li, Zhiyuan Tang, Xiang Sun \\ Hebei University of Engineering College of Information \& Electronic Engineering, Handan, China \\ E-mail: zjijun@gmail.com,lihua9896@163.com \\ Received May 16, 2010; revised May 27, 2010; accepted June 1, 2010
}

\begin{abstract}
Localization technology is an important support technology for WSN(Wireless Sensor Networks). The centroid algorithm is a typical range-free localization algorithm, which possesses the advantages such as simple localization principle and easy realization. However, susceptible to be influenced by the density of anchor node and uniformity of deployment, its localization accuracy is not high. We study localization principal and error source of the centroid algorithm. Meanwhile, aim to resolve the problem of low localization accuracy, we proposes a new double-radius localization algorithm, which makes WSN node launch periodically two rounded communications area with different radius to enable localization region to achieve the second partition, thus there are some small overlapping regions which can narrow effectively localization range of unknown node. Besides, partition judgment mechanism is proposed to ascertain the area of unknown node, and then the localization of small regions is realized by the centroid algorithm. Simulation results show that the algorithm without adding additional hardware and anchor nodes but increases effectively localization accuracy and reduces the dependence on anchor node.
\end{abstract}

Keywords: Wireless Sensor Networks; Localization Technology; Centroid Algorithm; Double-Radius Localization Algorithm; Partition Judgment Mechanism

\section{Introduction}

Localization technology, the premise to realize related applications from determining the localization of the incident or locking access to the node localization of the message by wireless sensor network, is a key support technology in WSN. Node localization information plays an important part in the target tracking, auxiliary routing, network management and many other areas [1]. There are two types of localization algorithms in localization technology, range-based and range-free localization algorithm. In recent years, with the obvious advantages, range-free localization algorithm has attracted much attention, meanwhile the centroid algorithm is a typical range-free localization algorithm which makes full use of the connectivity of network to locate with the obvious advantages in simple localization principle, little power consumption and easy realization. But its localization error has been prone to influence by the issues such as laying uniformity of anchor node and density of anchor nodes $[1,2]$. In order to solve the problem, the article studies centroid algorithm principle and error sources of localization and proposes double-radius algorithm based on partition judgment mechanism. The algorithm without adding additional hardware and anchor nodes but increases effectively localization precision and reduces the dependence on anchor nodes.

\section{The Centroid Algorithm and Error Analysis}

\subsection{Research on the Centroid Algorithm}

In the WSN localization technology, the range-free localization algorithm with the obvious advantages has been researched by lots of scholars. Due to its unique characteristics and advantages, the centroid localization algorithm is simple in principle with easy realization as well as little communication expense, so the typical range-free localization algorithm has get more concerned.

In [3], the enhanced centroid localization algorithm was proposed. The algorithm, by using more anchor nodes localization information indirectly from the infor- 
mation exchange with unknown neighbor nodes, with the addition of a small amount of traffic circumstances, made the localization accuracy of anchor node randomly distributed improved greatly and provided a new option for node localization in WSN. Furthermore, some researchers proposed decentralized intensity-weighted multi-hop field centroid localization algorithm improving localized nodes ratio by multi-hop centroid calculating and localization accuracy by decentralizing process and signal strength-weighting process. Compared with the original centroid algorithm, the average localization error of the algorithm can be reduced by half or so, and the localized ratio under the circumstance of low node density is close to 1 [4].

The paper studies the centroid algorithm from the view of division judgment in communication district. Firstly, fundamental questions such as localization principle, characteristics and error source of localization are researched. Then, double-radius localization algorithm based on partition judgment mechanism is improved to solve low localization accuracy.

\subsection{The Localization Principle and the Pros and Cons of the Centroid Algorithm}

The basic idea of the centroid algorithm is that, using the geometric centroid to its own estimated localization which is covered by unknown node and the polygon which composed of overlay area of beacon node's communication area in the communication range of the unknown node. Centroid is the geometric center of a polygon. The average of each vertex coordinates in a polygon is the coordinate of centroid. The centroid algorithm defines the district containing unknown node firstly, namely the polygon containing unknown node, then calculates the centroid of the polygon which is the localization of the unknown node.

In the centroid algorithm, anchor nodes periodically broadcast beacon packets which contain anchor node identification number and localization information to the neighbor nodes. When the unknown node receives the amount of beacon group from different anchor nodes exceeds a certain threshold $k$ or achieves a certain localization accuracy, it will define the centroid of polygon consisted by these node as its localization.

$$
\left(X_{e s t}, Y_{e s t}\right)=\left(\frac{X_{i 1}+\ldots \ldots+X_{i k}}{k}\right),\left(\frac{Y_{i 1}+\ldots \ldots+Y_{i k}}{k}\right),
$$

$\left(X_{i 1}, Y_{i 1}\right) \ldots\left(X_{i k}, Y_{i k}\right)$ is the unknown node can receive the anchor node coordinates information $[5,6]$.

The centroid localization algorithm is simple in principle, easy to algorithm realization with small amount of calculation, without additional hardware, fully Web-based connectivity, and without coordination between anchor nodes and unknown nodes [7].

However, centroid is the estimate as the localization of practical node, the accuracy and anchor node density of this estimate is related greatly with the evenness of node distribution, the bigger the density, more uniform of distribution, and the higher localization accuracy. Besides, the estimated is also related to the regular level of node's wireless signal propagation model, the closer to ball style, the higher degree.

\subsection{Error Analysis $[8,9]$}

Through the above analysis on localization principle of the centroid algorithm shows that the localization error of the centroid algorithm has the following four main sources. Take three anchor nodes to locate an unknown node as an example.

1) Centroid is the estimate as the localization of practical node

In the centroid algorithm, according to the connectivity among nodes can determine the region of unknown node, as shown in Figure 1. It can be defined that node $\mathrm{N}_{1}$ is inside overlap region $\mathrm{A}, \mathrm{B}, \mathrm{C}$ of three circles, but it can tell detailed localization of the node. The method that the algorithm defined the geometric centre $\mathrm{N}_{0}$ of overlapping region polygon as the actual localization of unknown node $\mathrm{N}_{1}$ is an estimate.

2) Unreasonable distribution of anchor nodes leading to big communication overlap region brings about the error

In the centroid algorithm, the localization accuracy is impacted by of anchor nodes distribution. When the anchor nodes are closer to the communication region of unknown node and the distribution are more uniform, the communication overlapping regions are smaller with more accurate localization. However, sometimes it is impacted by environmental constraints, so the node cannot be distributed ideally, but with random laying nodes. Nevertheless, some nodes have a greater localization error. As shown in Figure 2, ABC are communication overlapping regions of three anchor nodes, where $N_{1}$ is the actual localization of unknown nodes, $\mathrm{N}_{0}$ is the node localization to be obtained. When the anchor nodes A, B and $\mathrm{C}$ are laid closely, overlapping regions is too large

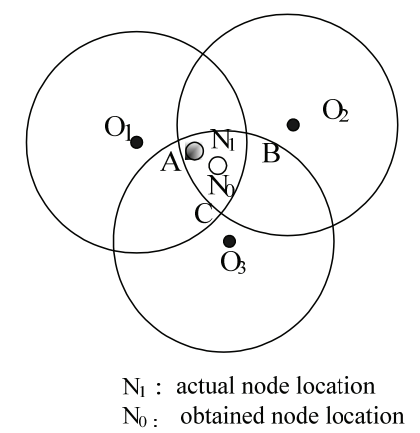

Figure 1. Localization diagram of the centroid algorithm. 


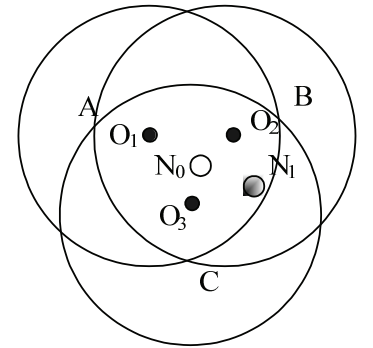

$\mathrm{N}_{1}$ : actual node location

$\mathrm{N}_{0}$ : obtained node location

Figure 2. Localization diagram of the centroid algorithm.

resulting in large localization errors.

3) The error due to a small amount of anchor node

The localization accuracy is impacted by density of unknown node' neighbor nodes, the greater the density, the smaller the overlap area of each anchor node' communication region, thus with a higher localization accuracy. With the less amount of anchor node, the overlap of communication region is bigger, thus with higher localization error. As Figure 3 shows that range of overlap region is larger with just two anchor nodes, so the localization error is higher.

4) Communication regions are not regular spheroid

The centroid algorithm localization accuracy is impacted by the degree of regular level of node's wireless signal propagation model. As Figure 4 shows, the communication regions formed by the wireless signal emitted from node is not a regular spheroid, the more regular the shape, the smaller the error.

\section{Double-Radius Localization Algorithm}

\subsection{Algorithm Idea}

From the above analysis, we can see that the centroid algorithm is using the overlap part of beacon node's communication area to determine the unknown node areas and calculate the area coordinate of centroid, the more number of anchor nodes, the more communication area, when the overlap part is more smaller, the localization is more precise, so reducing the scope of overlap part can improve the localization accuracy. Increasing the anchor nodes can improve the localization accuracy, but it will increase input costs, it will be the research focus that how to achieve multiple communications areas without increasing anchor node and thus getting as small as possible overlap of communication area.

We proposes double-radius algorithm based on partition judgment mechanism, the basic idea is that anchor node transmits periodically different power signals to produce two rounded communications area with different radius, and then realizes a number of overlapping com-

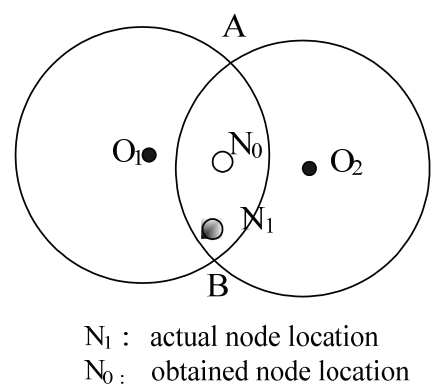

Figure 3. Localization diagram of the centroid algorithm.

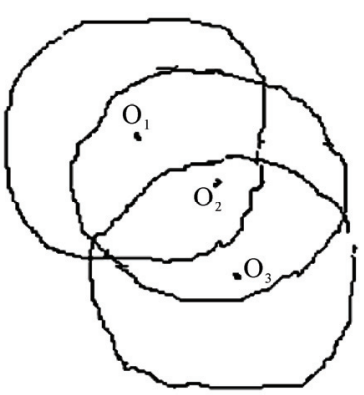

Figure 4. Localization diagram of the centroid algorithm.

munication area without increasing the case of anchor node, it can get a smaller communication overlap part, just as a secondary partition in the region.

Double-radius localization algorithm uses the two communication area with different radius which is launched by node to achieving partition, if the partitions are uneven and thereby causing occurrence of a larger localization region, but the smaller partition will be good for localization. The localization results would be influenced by the ascertainment of the small radiuses and its relation with big radius which are related to actual application and layout of anchor nodes. The aim is to achieve uniform partition to avoid large overlap.

The centroid algorithm and dual-radius localization mechanism will be compared from a qualitative point of view to show advantages of dual-radius localization algorithm. Shown in Figure 5, one solid circle is the node communication radius under high-power and dashed circle is the node communication radius under low power. In Figure 5(a), the centroid algorithm is used to locate unknown node $\mathrm{N}_{1}$, the result is the centroid $\mathrm{N}_{0}$ of regional BDF, showing a large error. When calculating the node $\mathrm{N}_{2}$, the centroid of overlap region ABDF, there is a serious error. In Figure 5(b), using double-radius localization mechanism to divide the region ABCDEF into 19 small regions, then using partition judgment to ascertain the smallest region, then calculating centroid of the region, we can obtain that the centroid of region 11 is coordinate of node $\mathrm{N}_{1}$ and the centroid of region 1 is coordinate of $\mathrm{N}_{2}$. Obviously, error of double-radius lo- 


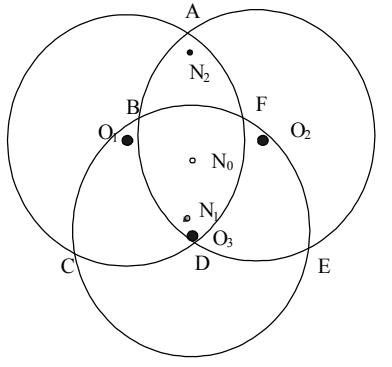

(a)

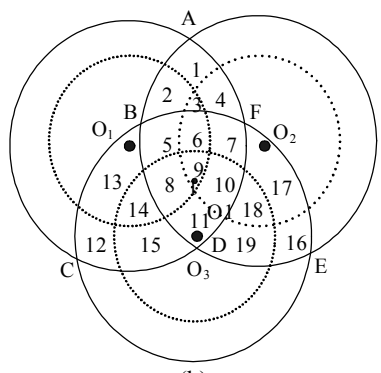

(b)
$\mathrm{N}_{0}$ : Obtained node location $0_{1}, 0_{2}, 0_{3}$ : Anchor node

Figure 5. Comparison of the centroid algorithm and doubleradius algorithm.

calization algorithm is smaller than error of centroid.

It will be seen that the double-radius localization algorithm can ascertain unknown node in a small region without increasing anchor node and additional hardware. Centroid coordinate, which considered unknown node coordinate, is obtained using the centroid algorithm in the region.

\subsection{Double-Radius Localization Algorithm Design}

It was shown in Figure 5, nodes transmit signal periodically with different power, appearing alternately two different radius of communication area, communications radius under high-power is $\mathrm{R}(\mathrm{m})$, the communications radius under low-power is $\mathrm{r}(\mathrm{m})$, the coordinates of $\mathrm{O}_{1}$ is $\left(\mathrm{X}_{1}, \mathrm{Y}_{1}\right)$, the coordinates of $\mathrm{O}_{2}$ is $\left(\mathrm{X}_{2}, \mathrm{Y}_{2}\right)$, the coordinates of $\mathrm{O}_{3}$ is $\left(\mathrm{X}_{3}, \mathrm{Y}_{3}\right)$. Communication regions under different power correspond to the different equation of circle. Meanwhile, both of the two signals have the ID of node, the equation message of the two circles and the different mark, which is easy to receive node to discern it.

The equation of the circle which is corresponded by node $\mathrm{O}_{1}, \mathrm{O}_{2}, \mathrm{O}_{3}$ is as follows:

$$
\begin{array}{ll}
\mathrm{O}_{1} & R^{2}=\left(X-X_{1}\right)^{2}+\left(Y-Y_{1}\right)^{2} \\
& r^{2}=\left(X-X_{1}\right)^{2}+\left(Y-Y_{1}\right)^{2} \\
\mathrm{O}_{2} & R^{2}=\left(X-X_{2}\right)^{2}+\left(Y-Y_{2}\right)^{2} \\
& r^{2}=\left(X-X_{2}\right)^{2}+\left(Y-Y_{2}\right)^{2} \\
\mathrm{O}_{3} & R^{2}=\left(X-X_{3}\right)^{2}+\left(Y-Y_{3}\right)^{2} \\
& r^{2}=\left(X-X_{3}\right)^{2}+\left(Y-Y_{3}\right)^{2}
\end{array}
$$

\section{Partition Judgment Mechanism}

Partition judgment mechanism is a method to define the region of unknown node according to the signal received by it. As shown in Figure 5, a communication region is divided into 19 small regions; through partition judgment mechanism to get the location of node. The followings are the judgment methods to define the region of the unknown node according to the different signals it receives.

1) When the node only receives the high-power communication signals from node $\mathrm{O}_{1}$ and node $\mathrm{O}_{2}$, using its corresponding Equations (1-4) reaches the equation of intersection regions; the region is shown in Figure 1, thus using the centroid algorithm to calculate the centroid of the small region.

$$
\left\{\begin{array}{l}
R^{2}>\left(X-X_{1}\right)^{2}+\left(Y-Y_{1}\right)^{2} \\
r^{2}<\left(X-X_{1}\right)^{2}+\left(Y-Y_{1}\right)^{2} \\
R^{2}>\left(X-X_{2}\right)^{2}+\left(Y-Y_{2}\right)^{2} \\
r^{2}<\left(X-X_{2}\right)^{2}+\left(Y-Y_{2}\right)^{2}
\end{array}\right.
$$

2) When the node only receives the power signal of the size $\mathrm{O}_{1}, \mathrm{O}_{2}$ high-power signals and $\mathrm{O}_{3}$ high-power signal, using the corresponding Equations $(2,4-6)$ to ascertain the region in Figure 5.

$$
\left\{\begin{array}{l}
r^{2}>\left(X-X_{1}\right)^{2}+\left(Y-Y_{1}\right)^{2} \\
R^{2}>\left(X-X_{2}\right)^{2}+\left(Y-Y_{2}\right)^{2} \\
r^{2}<\left(X-X_{2}\right)^{2}+\left(Y-Y_{2}\right)^{2} \\
R^{2}>\left(X-X_{3}\right)^{2}+\left(Y-Y_{3}\right)^{2} \\
r^{2}<\left(X-X_{3}\right)^{2}+\left(Y-Y_{3}\right)^{2}
\end{array}\right.
$$

3) When the node only receives the low-power signals $\mathrm{O}_{1}, \mathrm{O}_{2}$, and high-power signals $\mathrm{O}_{3}$, using the corresponding Equations $(2,4-6)$ to concludes the region 6 .

$$
\left\{\begin{array}{l}
r^{2}>\left(X-X_{1}\right)^{2}+\left(Y-Y_{1}\right)^{2} \\
r^{2}>\left(X-X_{2}\right)^{2}+\left(Y-Y_{2}\right)^{2} \\
R^{2}>\left(X-X_{3}\right)^{2}+\left(Y-Y_{3}\right)^{2} \\
r^{2}<\left(X-X_{3}\right)^{2}+\left(Y-Y_{3}\right)^{2}
\end{array}\right.
$$

4) When the node only receives the $O_{1}$ power signal, $\mathrm{O}_{3}$ power signal and $\mathrm{O}_{2}$ high-power signals, using the corresponding Equations $(2-4,6)$ to conclude the region 8 .

$$
\left\{\begin{array}{l}
r^{2}>\left(X-X_{1}\right)^{2}+\left(Y-Y_{1}\right)^{2} \\
r^{2}>\left(X-X_{3}\right)^{2}+\left(Y-Y_{3}\right)^{2} \\
R^{2}>\left(X-X_{2}\right)^{2}+\left(Y-Y_{2}\right)^{2} \\
r^{2}<\left(X-X_{2}\right)^{2}+\left(Y-Y_{2}\right)^{2}
\end{array}\right.
$$

5) When the node only receives the $\mathrm{O}_{1}$ low-power signal, $\mathrm{O}_{2}$ low-power signal and $\mathrm{O}_{3}$ low-power signal, using 
the corresponding Equations $(2,4,6)$ to conclude the region 9.

$$
\left\{\begin{array}{l}
r^{2}>\left(X-X_{1}\right)^{2}+\left(Y-Y_{1}\right)^{2} \\
r^{2}>\left(X-X_{2}\right)^{2}+\left(Y-Y_{2}\right)^{2} \\
r^{2}>\left(X-X_{3}\right)^{2}+\left(Y-Y_{3}\right)^{2}
\end{array}\right.
$$

6) When the node only receives the $\mathrm{O}_{1}$ high-power signals, $\mathrm{O}_{2}$ high-power signals, and $\mathrm{O}_{3}$ low-power signal, using the corresponding Equations $(1-4,6)$ to conclude the region 11 .

$$
\begin{aligned}
& R^{2}>\left(X-X_{1}\right)^{2}+\left(Y-Y_{1}\right)^{2} \\
& r^{2}<\left(X-X_{1}\right)^{2}+\left(Y-Y_{1}\right)^{2} \\
& R^{2}>\left(X-X_{2}\right)^{2}+\left(Y-Y_{2}\right)^{2} \\
& \mathrm{r}^{2}<\left(X-X_{2}\right)^{2}+\left(Y-Y_{2}\right)^{2} \\
& r^{2}>\left(X-X_{3}\right)^{2}+\left(Y-Y_{3}\right)^{2}
\end{aligned}
$$

7) When the node only receives the $O_{1}$ power signal and $\mathrm{O}_{2}$ high-power signals, using the corresponding Equations (1-4) to reach the intersection region 2, 5, 8 .

$$
\begin{aligned}
& r^{2}>\left(X-X_{1}\right)^{2}+\left(Y-Y_{1}\right)^{2} \\
& R^{2}>\left(X-X_{2}\right)^{2}+\left(Y-Y_{2}\right)^{2} \\
& r^{2}<\left(X-X_{2}\right)^{2}+\left(Y-Y_{2}\right)^{2}
\end{aligned}
$$

8) When the node only receives the $\mathrm{O}_{1}$ power signal and $\mathrm{O}_{2}$ power signal, using the corresponding Equations $(2,4)$ to reach the intersection region $3,6,9$.

$$
\begin{aligned}
& r^{2}>\left(X-X_{1}\right)^{2}+\left(Y-Y_{1}\right)^{2} \\
& r^{2}>\left(X-X_{2}\right)^{2}+\left(Y-Y_{2}\right)^{2}
\end{aligned}
$$

The remaining region and the solved region have a symmetrical relationship, so they can be obtained as above.

In article, it takes that an unknown node can be located with three anchor nodes as example. In the case of multiple anchor nodes, an unknown node can be located. That is, the unknown node uses the communication region equation of all the anchor nodes received, it determines the unknown node's location according to partition judgment mechanism, and then uses the existing the centroid algorithm to get the centroid of this region.

\section{Algorithm Simulation}

In this simulation we use MATLAB, and the basic idea is: Firstly, we simulate the location environment and motion tracks of the node, use dual-radius algorithm and the centroid algorithm to derive moving nodes' theoretical trajectories respectively, and then compare their fitting degree intuitively. Secondly, using the two algorithms to calculate the location at every $20 \mathrm{~m}$ respectively, get the two theoretical error value of localization, thus derive the average value, and finally compare the average error value of the centroid algorithm and dual-radius localization error algorithm.

It is shown in Figure 6, we select three anchor nodes randomly, these are the black point positions at the center of these circles, here we assume the coordinates: $(0,0),(-45,45 \sqrt{3}),(45,45 \sqrt{3})$, and the big circle radius: $90 \mathrm{~m}$, the smaller one: $60 \mathrm{~m}$, then verify our algorithm in several instances which select quadratic spline curve to simulate the moving target. In Figure 6, black spots with crosses are the centroid of overlapping regions; the compacting curve is the actual trajectory of the node to be tracked, the dashed line is the virtual trajectory which is measured by these two algorithms respectively. The closer the two curves are, the more accurate positioning we did, we can see that the curve which corresponds to dual-radius algorithm is closer to the node's actual trajectory.

As described above, we select 9 positions as the abscissa axis in Figure 6, and the negative sign on the opposite direction. We figure out theoretical locations of these none nodes through the centroid algorithm and the dual-radius algorithm respectively. Figure 7 is the error line graph for algorithms, in which thick lines is the error curve for double-radius algorithm, and the thin one is for the centroid algorithm, because of their symmetry relations respectively, we get positioning error values for the 5 position, as shown in Table 1.

Data show that, the average error and accuracy of double-radius algorithm are $3.26 \mathrm{~m}$ and $3.62 \%$ respectively. However, the parameters of the centroid algorithm are $9.46 \mathrm{~m}$ and $10.51 \%$. Obviously, dual-radius algorithm has a higher positioning accuracy, reducing the dependence on the anchor node, reaching a large extent better than the centroid algorithm, and is able to meet the needs of the usual location when there are no additional anchor nodes and no extra hardware resources. Dual-radius algorithm also has its own inadequacies, it requested the node periodically launching two different power signals in order to determine the areas that the unknown node to be located, and the algorithm power is slightly higher than the centroid algorithm power.

\section{Conclusions}

Localization accuracy of the centroid algorithm is low but related to neighbors' density of anchor node and distribution evenness. In the practical application, the algorithm is constrained by the density and distribution of node without ideal localization accuracy.

To solve the above problems, the paper proposes double-radius localization algorithm based on partition judg- 


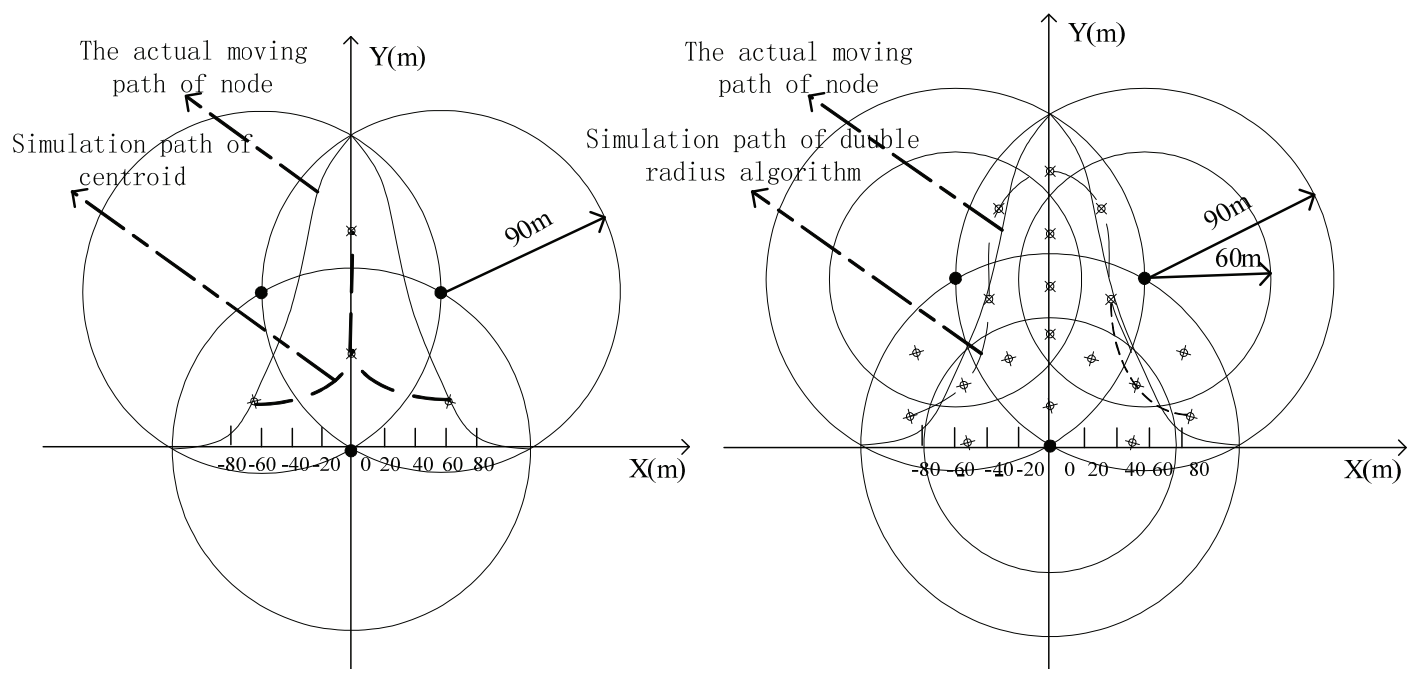

Figure 6. Comparison of the centroid algorithm and double-radius algorithm, (a) path simulation grath of centroid algorithm; (b) path simulation grath of double radius algorithm.

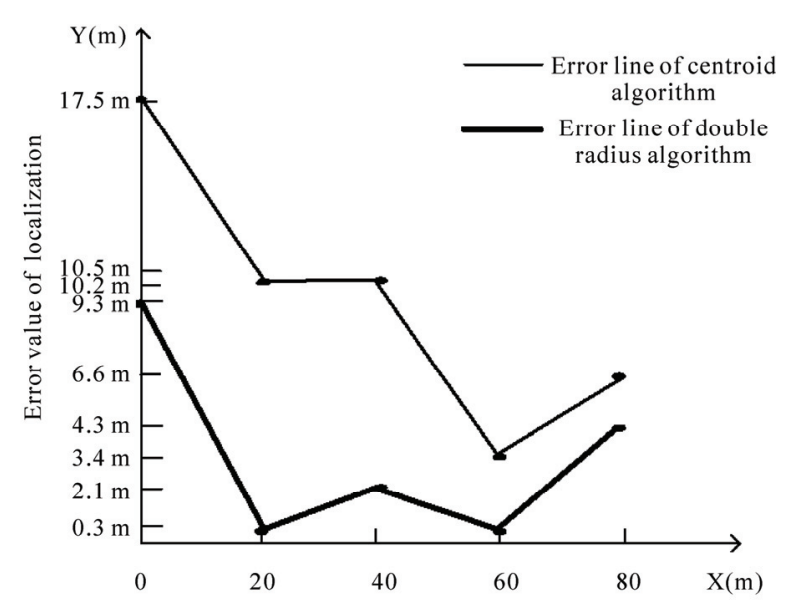

Figure 7. Line graph of algorithm error.

Table 1. Data table of algorithm error.

\begin{tabular}{cccccccc}
\hline $\begin{array}{c}\text { Location } \\
\text { Point }\end{array}$ & $0 \mathrm{~m}$ & $20 \mathrm{~m}$ & $40 \mathrm{~m}$ & $60 \mathrm{~m}$ & $80 \mathrm{~m}$ & average accuracy \\
\hline Centroid & 17.5 & 10.2 & 10.5 & 3.4 & 6.6 & 9.46 & $10.51 \%$ \\
$\begin{array}{c}\text { Double- } \\
\text { Radius }\end{array}$ & 9.3 & 0.3 & 2.1 & 0.3 & 4.3 & 3.26 & $3.26 \%$ \\
\hline
\end{tabular}

ment mechanism, it launches different power signals periodically through the WSN node, so that node has two different alternating radius communication regions. As the communication region is divided twice, there has been a number of small communication overlapping regions. Then the specific area of unknown node can be determined by using the above mechanism. Simulation results show that the algorithm relative localization error has been reduced from $10.51 \%$ to $3.62 \%$, but without additional anchor and hardware.

\section{References}

[1] F. B. Wang, L. Shi and F. Y. Ren, "Self-Localization Systems and Algorithms for Wireless Sensor Networks," Chinese Journal of Software, Vol. 16, No. 5, 2005, pp. 857-868.

[2] N. Bulusu, J. Heidemann and D. Estrin, "GPS-Less Low Cost Outdoor Localization for Very Small Devices," IEEE Personal Communications Magazine, Vol. 7, No. 5, 2000, pp. 28-34.

[3] Z. B. Li, Z. Z. Wei and F. L. Xu, "The Performance Analysis of Advanced Centroid Localization Algorithm for Wireless Sensor Network," Chinese Journal of Sensors and Actuators, Vol. 22, No. 4, 2009, pp. 563-566.

[4] X. An, T. Jiang and Z. Zou, "Centroid Localization Algorithm for Wireless Senor Network," Computer Engineering and Applications, Vol. 43, No. 20, 2007, pp. 136-138.

[5] Rudafshanim and S. Datta, "Localization in Wireless Ad Hoc Sensor Networks," International Conference on Information Processing in Sensor Networks, Cambridge, 2007, pp. 51-60.

[6] T. He, C. D. Huang and B. M. Blum, "Range-Free Localization Schemes in Large Scale Sensor Networks," Proceedings of the 9th Annual International Conference on Mobile Computing and Networking, New York, 2003, pp. 81-95.

[7] Y. Qiu, C. C. Zhao, G. Dai and C. J. Hu, "Research on Localization Technology for Wireless Sensor Networks, Computer Science, Vol. 5, No. 35, 2008, pp. 47-50.

[8] L. Gao, X. Q. Zheng and H. Zhang, "A Node Localization Algorithm for Wireless Sensor Network Based on Trilateration and Centroid Algorithm," Journal of Chongqing Institute of Technology, Vol. 23, No. 7, 2009, pp. 138-141.

[9] Y. G. Cheng and H. Xu, "Hops-Weighted Centroid Localization Algorithm for Wireless Sensor Network," Computer Engineering and Applications, Vol. 45, No. 7, 2009, pp. 105-107. 